\title{
Present and future Chinese large telescope projects
}

\author{
Xiangqun Cui \\ National Astronomical Observatories \\ Nanjing Institute of Astronomical Optics and Technology, CAS \\ 188 Bancang Street, Nanjing 210042, \\ P. R. China \\ email: xcui@niaot.ac.cn
}

\begin{abstract}
This report is a general introduction on Chinese large telescope projects. It includes the ongoing project Large Sky Area Multi-Object Fiber Spectroscopic Telescope (LAMOST), and three projects which have reviewed by Chinese government recently: Five-hundred-meter Aperture Spherical (radio) Telescope (FAST); Space Solar Telescope (SST); Hard X-ray Modulation Telescope (HXMT). These three projects have finished their feasibility studies and development of key technologies. They are very likely to be approved by the Chinese government in 2006. Besides these large telescope projects, the site survey in Western China for large telescopes in optical, infrared, sub-millimeter and millimeter astronomy, the preliminary study on Chinese future giant optical/infrared telescopes, and a future extremely large wide field telescope are also briefly introduced.
\end{abstract}

Keywords. Large ground based telescope, Space telescope, Site survey

\section{Introduction}

Many astronomical facilities have been developed in China in the last several decades. The rapid development of the Chinese economy in the past ten years has contributed to a rapid increase of investment in science and education in China. Chinese astronomy has started to enter a golden period. In addition to a new Chinese-made $1 \mathrm{~m}$ near-Earth object survey telescope, which has been installed at the Xuyi Station of the Purple Mountain Observatory, two 50m radio telescopes and a $1 \mathrm{~m}$ infrared solar telescope are under construction, and a UK-made $2.4 \mathrm{~m}$ optical telescope is now in its installation stage at Gaomeigu Station of the Yunnan Observatory. The most exciting project in Chinese astronomy is the Large Sky Area Multi-Object Fiber Spectroscopic Telescope (LAMOST), which has started installation on site now. The Space Solar Telescope (SST), the Hard X-ray Modulation Telescope (HXMT) and the Five-hundred-meter Aperture Spherical (radio) Telescope (FAST) have had about ten years of pre-study on scientific goals and key techniques. We are hopeful that all these projects will be approved as National large scientific projects soon.In addition, the NAOC has initialized a site survey in the vast area of Western China, in order to find potential sites for a future giant ground-based optical/IR telescope, sub-millimeter- and millimeter-wave telescopes. By 2012, most of the above projects will be either completed or near completion, while the future giant ground-based optical/IR telescope and sub-millimeter-wave telescope projects will most likely have commenced. In this paper a general and brief introduction on these large telescope projects, the site survey in Western China and the preliminary study on future large optical/IR telescope are given. 


\section{Large Sky Area Multi-Object Fiber Spectroscopic Telescope (LAMOST)}

LAMOST is a meridian reflecting Schmidt telescope with an average clear aperture of 4 meter, a focal length of 20 meter and a field of view of 5 degree (Wang et al. 1996, $\mathrm{Su}$ et al. 1998). It is laid down on the ground with its optical axis fixed in the meridian plane and consists of a reflecting Schmidt corrector $\mathrm{M}_{\mathrm{A}}$ at the Northern end, a spherical primary mirror $\mathrm{M}_{\mathrm{B}}$ at the Southern end and a focal surface in between (Fig. 1). Both the primary mirror and the focal surface are fixed on their ground bases, and the reflecting corrector, as a coelostat, tracks the motion of celestial objects. Celestial objects are observed around their meridian passages. The light collected is reflected from $M_{A}$ to $\mathrm{M}_{\mathrm{B}}$, and then reflected by $\mathrm{M}_{\mathrm{B}}$ and forms image of the observed sky on the focal surface. The light of individual objects is fed into the front ends of 4000 optical fibers accurately positioned on the focal surface and then transferred into the 16 spectrographs fixed in the room underneath to be dispersed into spectra and recorded on $324 \mathrm{k} \times 4 \mathrm{k}$ CCD detectors simultaneously.

The site of LAMOST is Xinglong station located at about 180km North of Beijing. The observing sky area is $-10^{\circ} \leqslant \delta \leqslant+90^{\circ}$ (total 24,000 square degrees). The spectral resolution is $1000 / 2000$ for low and 5000/10000 for medium settings. If taking spectral resolution $1 \mathrm{~nm}$, the survey capability maximum magnitude is $20.5^{\mathrm{m}}$ at Xinglong station with about 1.5 hours integration time. LAMOST is designed for wide field and large sample astronomy. Its main scientific goals are (1) extragalactic spectroscopic survey large-scale structure of the universe and physics of galaxies, (2) stellar spectroscopic survey - structure of the Galaxy and stellar astrophysics, and (3) cross identification of multi-wave band surveys.

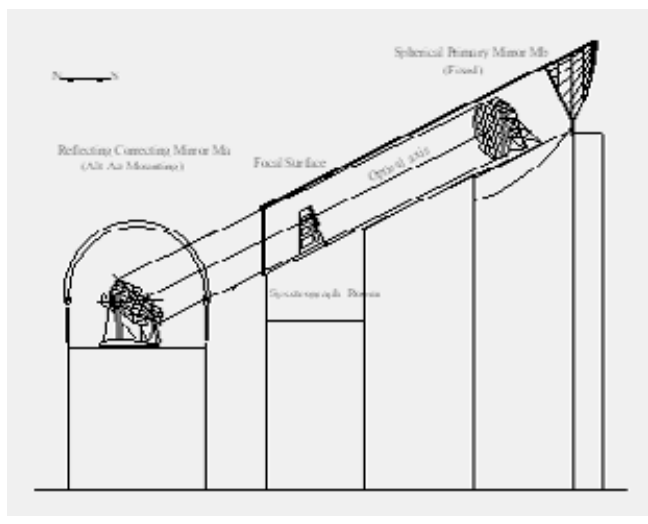

Figure 1. Overview of LAMOST.

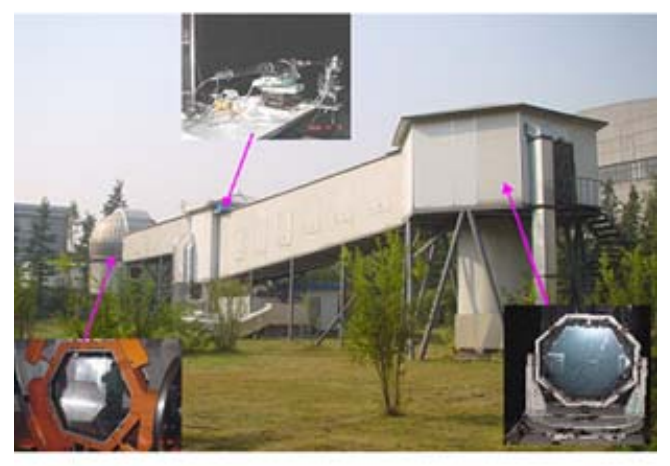

Figure 2. 1m LAMOST for experiment of active optics.

LAMOST is a novel telescope with some new concepts and new technologies especially in the following aspects: (a) large field of view with large aperture; (b) use of active optics to realize an optical system which could not be realized by the conventional optical system, (c) a variable large aspheric mirror surface by combining active optics technologies in both segmented mirror and thin deformable mirror, (d) Spectroscopy with 4000 parallel controllable fibers on a partitioned focal surface.

A $1 \mathrm{~m}$ LAMOST has been built for the active optics experiment (Fig. 2) with a full scale but with only one sub-mirror of each mirror (Su \& Cui 2004, Cui et al. 2004). The best results from the close loop active correction experiment with the autocollimation mode 
is $80 \%$ energy circled $\left(\mathrm{d}_{80}\right)$ in 0.52 arcsec for image quality. For the close loop correction with the real time observing mode, in about 2 hours tracking a star, the average corrected image quality is $\mathrm{d}_{80}$ in 1.505 arcsec. The open loop correction is the most difficult case. In about 4 hours tracking, the average result we got from the open loop correction is $\mathrm{d}_{80}$ in 1.6 arcsec.

Prototypes of the optical fiber positioning system and spectrograph are completed also and the formal ones are in manufacturing. Now the installation has been started on site and the expected first light will be in 2007 .

\section{Five-hundred-meter Aperture Spherical (radio) Telescope (FAST)}

The Five hundred meter Aperture Spherical Telescope (FAST) is an Arecibo type large radio telescope (Fig. 3) and is also a Chinese concept for SKA (Nan \& Peng 2000). FAST is proposed to be built in the unique karst area of Southwest China. It will be over twice as large as Arecibo coupled with much wider sky coverage. Technically, FAST is not simply a copy of the existing Arecibo telescope but has rather a number of innovations: (1) the proposed main spherical reflector, by conforming to a paraboloid of revolution in real time through actuated active control, enables the realization of both wide bandwidth and full polarization capability while using standard feed design; (2) a feed support system, which integrates optical, mechanical and electronic technologies, will effectively reduce the cost of the support structure and control system. The reflector is a dish with a diameter of $500 \mathrm{~m}$ and a radius of curvature $300 \mathrm{~m}$. The illuminated aperture is $300 \mathrm{~m}$. The opening angle is $120^{\circ}$. For the sky coverage, the maximum observing zenith angle is $50^{\circ}$. Its working frequencies are $0.1-2 \mathrm{GHz}$, up to $\mathrm{C}$-band and $\mathrm{X}$-band. The pointing accuracy is $4 "$ and the slewing speed is $10^{\circ}$ per minute.

FAST is a key project in the Chinese Academy of Sciences for many years. The prototype for the feed, the active panels and a $50 \mathrm{~m}$ prototype of FAST have been finished, and some experiments have been successfully done during recent years. In 2005, the FAST project has passed the review by the Chinese Academy of Sciences. Hopefully, it is going to be approved by the Chinese government in 2006 .

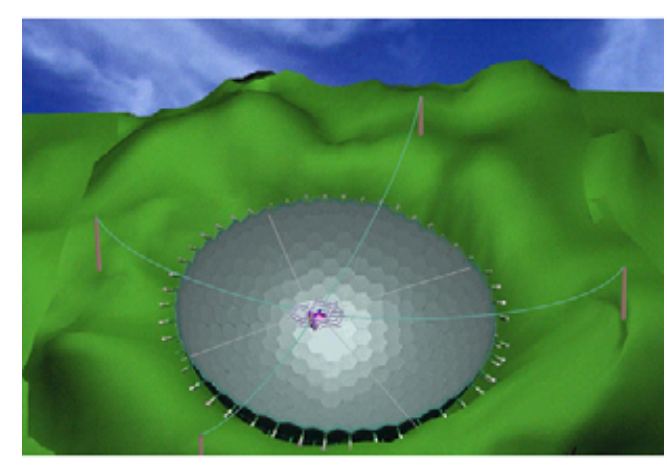

Figure 3. Five hundred meter Aperture Spherical Telescope (FAST).

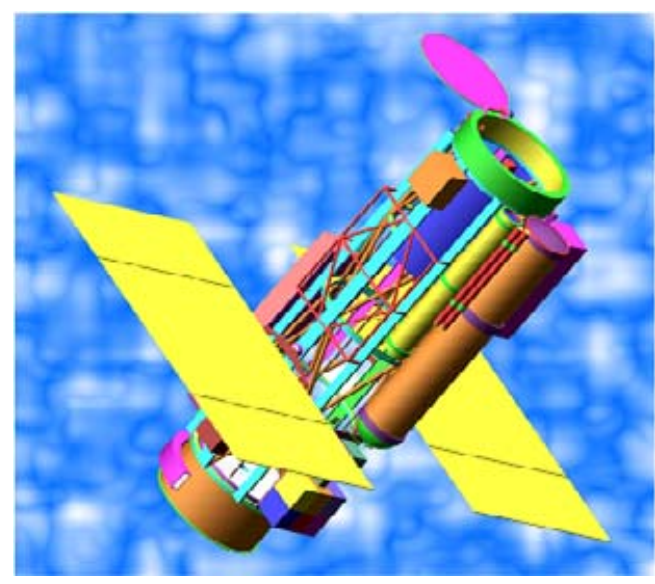

Figure 4. Space Solar Telescope (SST). 


\section{Space Solar Telescope (SST)}

Chinese astronomy has entered into the space era. The Space Solar Telescope (SST) is proposed to take the advantage of rocketry and abundant research work in solar magnetic field and velocity field in China. SST is a $1 \mathrm{~m}$ space solar telescope (Fig. 4) with diffraction limited optical image, equipped with an innovated 2-d spectrograph, a 0.5 arcsec high resolution soft X-ray telescope, and wide band spectrometer. It will be used in wide band, continuing evolution observation. With its main character of high resolution, and concentrated in detecting the magnetic element, the study and observation in the instant and stable magnetic hydrokinetics will take place. The total weight of SST will be $2000 \mathrm{~kg}$, orbit at altitude $709 \mathrm{~km}$, and three-axis stabilized. The data flow rate will be $60 \mathrm{Mb} / \mathrm{s}$, average power $1200 \mathrm{~W}$ and the mission lifetime 3 years. Until now, nearly all key technologies have been developed and tested.

\section{Hard X-ray Modulation Telescope (HXMT)}

The Hard X-ray Modulation Telescope (HXMT) has been selected recently as the first Chinese space astronomy mission for launch in 2010 (Fig. 5). Its main scientific goals are deep hard X-ray all-sky surveys between 20-250keV to discover about 1000 new supermassive black holes, pointed observations of faint objects, high sensitivity timing studies and high sensitivity detection of other hard X-ray sources. The characteristic are: (1) main detector - $\mathrm{NaI}(\mathrm{Tl}) / \mathrm{CsI}(\mathrm{Na})$ Phoswich; (2) total detect area — 5000 $\mathrm{cm}^{2} ;$ (3) energy range - $20 \sim 250 \mathrm{keV}$; (4) energy resolution — 22\% (at $60 \mathrm{keV}$ ); (5) continuum sensitivity - $\sim 3.0 \cdot 10^{-7}$ photons $\mathrm{cm}^{-2} \mathrm{~s}^{-1} \mathrm{keV}^{-1}\left(3 \sigma\right.$ at $100 \mathrm{keV}$ in $\left.10^{5} \mathrm{~s}\right)$; (6) field of view $-5.7^{\circ} \times 5.7^{\circ}(\mathrm{FWHM}) ;(7)$ source location $-1 \operatorname{arcmin}(20 \mathrm{~s})$; (8) angular resolution $-\leqslant 5$ arcmin $(20 \mathrm{~s}) ;(9)$ total mass about $1100 \mathrm{~kg}$ (payload $\sim 700 \mathrm{~kg}$ ); (10) dimension $-1.7 \mathrm{~m} \times 1.7 \mathrm{~m} \times 1.2 \mathrm{~m}(\mathrm{LWH}) ;(11)$ nominal mission lifetime -2 years; $(12)$ orbit altitude $-550 \mathrm{~km}$ and inclination $43^{\circ}$ attitude; (13) three-axis stabilized; (14) control precision $\pm 0.25^{\circ}(15)$ stability $0.005^{\circ} / \mathrm{s} ;(16)$ measurement accuracy $<0.01^{\circ}$. A laboratory prototype has been successfully developed and tested during last several years. Possible secondary instruments through international collaborations: (1) Soft X-ray telescope; (2) Wide field X-ray monitor, etc.

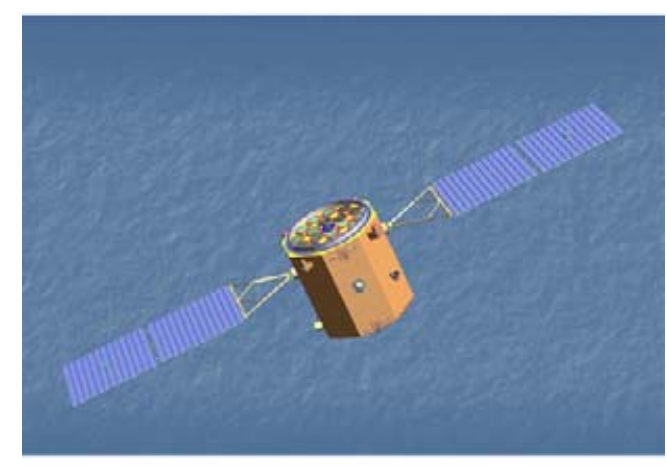

Figure 5. Hard X-ray Modulation Telescope (HXMT).

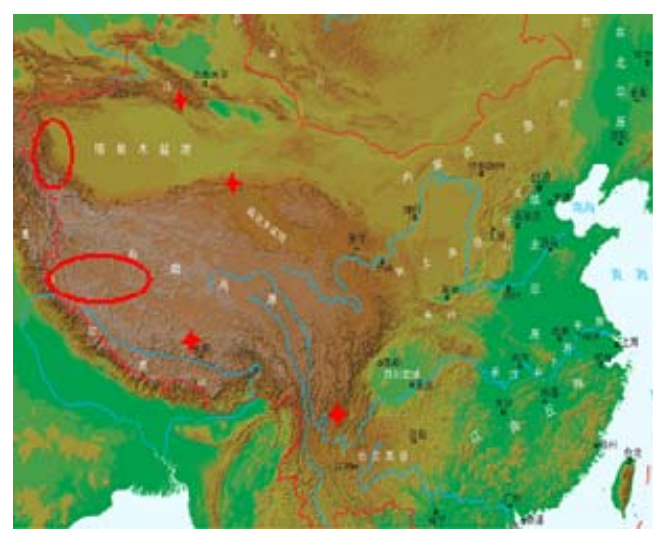

Figure 6. Potential sites in Western of China. 


\section{Site survey in west of China}

The Western China is a vast area (Fig. 6). Most places of this area are at high altitude, with clean air, low temperature, dry environment, little cloud cover and dark sky. Targeting sites for ELT (opt-IR-submm), the National Astronomical Observatories CAS decided to start the site survey in Western China (Tibet, Xinjiang, Qinghai, Yunnan) about three years ago. The plan for the site survey in Western China is ten years: 2 years for organization and instruments and 3 years thereafter $5 \sim 10$ candidate sites should be selected. After 5 years follows the monitoring of 2-3 sites for 2 years; before the second 5 years, the result review, the concept of a Chinese ELT and the final goal of site survey project will be settled down. In the second 5 years, a long-term monitoring with specific instruments and the final selection will be obtained. The site survey has set up stations in 2005 in the Pamir altiplano (April), Ali (July) and 4 reference stations including the instrumentation installation and the monitoring activity. The instrumentation has been ordered through international collaborations.

\section{Extremely large telescopes}

In 2000, the study group in Nanjing Institute of Astronomical Optics and Technology (NIAOT) for a preliminary study of an extremely large telescope was established. Since then, several configurations for extremely large telescopes have been put forward (Su et al. 2000, Su et al. 2004a, Su et al. 2004b). The recent configuration is a 30m optic/infrared telescope - the Chinese Future Giant Telescope (CFGT), and two configurations for an extremely large wide-field telescope. The potential sites are Western China and Antarctica (Dome A/C). For the Chinese Future Giant Telescope (Fig. 7), the characteristics are: (1) one primary focus with FoV $100 \%$ energy concentrated in diameter $\left(\mathrm{d}_{100}\right)$ in 0.36 arcsec in FoV 20 arcmin, 4 Nasmyth foci with $\mathrm{d}_{100} 0.086$ arcsec in FoV 8 arcmin (the diffraction limited in FoV 2.83 arcmin), one Cassegrain focus with $\mathrm{d}_{100} 0.11$ arcsec in FoV 8 arcmin (diffraction limited in FoV 2.17 arcmin), one Coude focus with FoV 29.59 arcsec (diffraction limited); (2) all with a Ritchey-Chré tien system; (3) aspherical primary mirror with F/1.2 and 1122 partial annular submirrors; (4) one secondary mirror with diameter $2.476 \mathrm{~m}$ for all optic systems (to exchange the optical system from two Nasmyth I to others, the displacements along optical axis, surface shape change of the secondary mirror and the tip-tilt of submirrors will be applied) or avoid the bigger surface change of the common secondary, the different secondary of different optic system is another option with diameter of all secondary mirrors smaller than $2.925 \mathrm{~m}$

For the extremely large wide field telescope, two configurations have been studied: (1) a LAMOST-type extremely large wide field telescope and (2) a new type extremely large wide field telescope. For both cases, all mirrors are plano and spherical. For the LAMOST-type extremely large wide field telescope, the Cassegrain focus is added. At the primary focal plane, the image quality with $\mathrm{d}_{80}$ less than 1 arcsec in FoV $3 \mathrm{deg}$ for a site in Antarctica, and $\mathrm{d}_{80}$ less than 0.16 arcsec in FoV 10 arcmin at Cassegrain focus. For a new type extremely large wide field telescope, the main innovation is to set up a corrector array at focal surface (Fig. 8) to correct the spherical aberration. The field of view depends on the area (also the number, if the aperture of each corrector is fixed), which should be compromised with the light obstruction area of the optical system. The image quality of each corrector (aperture $76 \mathrm{~mm}$ in diameter) is $\mathrm{d}_{80}<0.47$ arcsec in FoV 4.8 arcmin for flat focal plane. The study group is now still working on it, and the paper about this study is going to be published soon. 


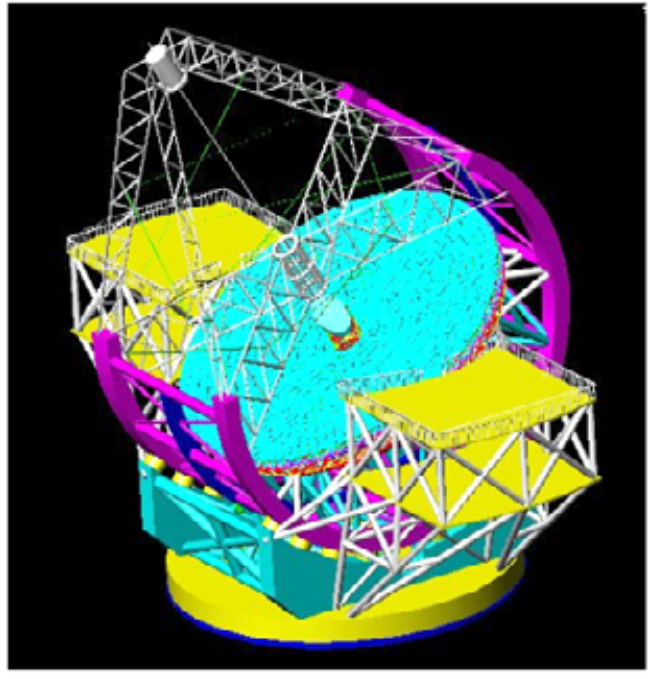

Figure 7. Concept design for Chinese future giant telescope.

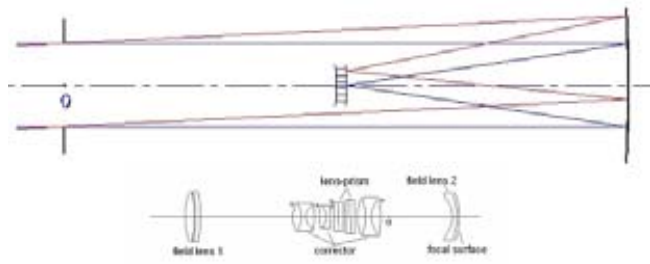

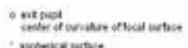

Figure 8. Corrector array for extremely large wide field telescope.

\section{Acknowledgement}

The author would like to thank Prof. Bo Peng, Prof. Suang Nan Zhang and Prof. Gang Zhao, for their help on information of FAST, HXMT, and site survey in Western China.

\section{References}

Cui, X.Q., Su, D.Q., Li, G.P., et al. 2004, SPIE 5489, 974

Nan, R.D. \& Peng, B. 2000, Acta Astronautica 46, 667

Su, D.Q., Cui, X.Q., Wang, Y.N. \& Yao, Z.Q. 1998, SPIE 3352, 76

Su, D.Q. \& Cui, X.Q. 2004, Chin. J. Astron. Astrophys. 4, 1

Su, D.Q., Cui, X.Q. \& Wang., Y.N. 2000, SPIE 4004, 340

Su, D.Q., Wang, Y.N. \& Cui, X.Q. 2004a, Chinese Astronomy and Astrophysics 28, 356

Su, D.Q, Wang, Y.N. \& Cui, X.Q. 2004b, SPIE 5489, 429

Wang, S.G., Su, D.Q., Chu, Y.Q., Cui, X.Q. \& Wang, Y.N. 1996, Appl. Opt. 35, 5155 